Cinémas

Revue d'études cinématographiques

Journal of Film Studies

\title{
Avec ou sans voix : la femme défaite
}

\section{Michèle Lagny}

Volume 16, numéro 1, automne 2005

Femmes et cinéma muet : nouvelles problématiques, nouvelles méthodologies

URI : https://id.erudit.org/iderudit/013053ar

DOI : https://doi.org/10.7202/013053ar

Aller au sommaire du numéro

Éditeur(s)

Cinémas

ISSN

1181-6945 (imprimé)

1705-6500 (numérique)

Découvrir la revue

Citer cet article

Lagny, M. (2005). Avec ou sans voix : la femme défaite. Cinémas, 16(1), 117-137. https://doi.org/10.7202/013053ar

\section{Résumé de l'article}

Le silence convient aux femmes : aussi l'écran muet donne-t-il une place généreuse à leurs corps et à leurs visages, magnifiés par la lumière. Paradoxalement, en Italie, on appelle les actrices les plus célèbres " divas ", tout comme les grandes cantatrices d'opéra. Ainsi le cinéma emprunte-t-il le vocabulaire d'un art où c'est la voix qui met les femmes en valeur, rehausse leur éclat ou amplifie leur défaite, les femmes étant le plus souvent sacrifiées à la fin d'un mélodrame, qu'il soit musical ou filmique. À travers certains rôles féminins de l'opéra du début $d u \mathrm{xx}^{\mathrm{e}}$ siècle, mis en scène par Puccini, et ceux du cinéma muet interprétés à la même époque par Francesca Bertini, l'auteure du présent article tente d'analyser les relations entre les corps de l'écran muet et les figures de la scène musicale. 


\section{Avec ou sans voix : la femme défaite}

\section{Michèle Lagny}

\section{RÉSUMÉ}

Le silence convient aux femmes: aussi l'écran muet donne-t-il une place généreuse à leurs corps et à leurs visages, magnifiés par la lumière. Paradoxalement, en Italie, on appelle les actrices les plus célèbres "divas", tout comme les grandes cantatrices d'opéra. Ainsi le cinéma emprunte-t-il le vocabulaire d'un art où c'est la voix qui met les femmes en valeur, rehausse leur éclat ou amplifie leur défaite, les femmes étant le plus souvent sacrifiées à la fin d'un mélodrame, qu'il soit musical ou filmique. À travers certains rôles féminins de l'opéra du début du $\mathrm{XX}^{e}$ siècle, mis en scène par Puccini, et ceux du cinéma muet interprétés à la même époque par Francesca Bertini, l'auteure du présent article tente d'analyser les relations entre les corps de l'écran muet et les figures de la scène musicale.

\section{ABSTRACT}

Silence suits women; further, silent film provides their bodies and faces with a luminous forum. Paradoxically, the most famous actresses in Italy are referred to as "divas" as one would of great operatic singers. As such, cinema borrows the parlance from an art form where voice defines their stature: accentuates their sizzle or emphasises their defeat. Also, whether musical or cinematic, women often are sacrificed to a melodrama's exigencies. Through selected operatic roles, as directed by Puccini at the beginning of the twentieth century, and those of silent film from the same period starring Francesca Bertini, the author strives herein to consider corporal figures from silent silver screen alongside those from the music scene. 
Peu à peu, m'apparaissent des mortes, des souffrantes, des déchirées; c'était comme un complot immense, venu du fond des âges, fait pour donner à voir ces femmes en proie à leur féminité, adorées et haïes, figures simulées d'une trop réelle société.

Clément 1979, p. 22

C'est l'impression laissée par le souvenir, ancien mais persistant dans ma mémoire, de la phrase placée ici en exergue et ayant pour objet des chanteuses d'opéra qui a orienté ma lecture des femmes sans voix des trente premières années du cinéma. Cinéma muet, donc. Les divas italiennes de cette époque restent pour nous des actrices toutes belles, talentueuses et capricieuses, "cent corps et cent visages d'un antique "star-system" du cinéma muet, à retrouver dans la splendeur de leur lumière unique " (Martinelli 2001, quatrième de couverture). Les divas occupaient pourtant deux lieux différents au début du $\mathrm{XX}^{\mathrm{e}}$ siècle: l'opéra, domaine du fantasme, scène déraisonnable où les mots ne se disaient qu'en sons musicaux; l'écran muet, où la parole absente était remplacée par la lumière. La question que cette "bipolarité " soulève est celle du rapport entre la mémoire musicale et la mémoire visuelle des images cinématographiques des divas, à la fois personnages, actrices, fantasmes; non pas «images» du "réel », mais figures, symboles du féminin qui, malgré tout, incarnent un certain nombre de réalités féminines.

Ce siècle, où s'épanouit l'opéra (en particulier en Italie) et où naît le cinéma (une forme d'art qu'on dit née au XX siècle, mais qui est en réalité profondément enracinée dans la culture et les représentations du XIX ${ }^{e}$ ), consacre "la primauté de la femme imaginaire»: "La femme est l'être qui projette la plus grande ombre et la plus grande lumière dans nos rêves ", écrivait Charles Baudelaire en 1861 dans Les paradis artificiels, texte repris en 1938 par André Breton et Paul Éluard dans le Dictionnaire abrégé du surréalisme (voir Michaud 1991, p. 125- 
145). Les héroïnes du mélodrame - tant musical, théâtral que filmique - sont des femmes composées d'ombre autant que de lumière, issues « du continent noir» et condamnées par la "fatalité " à l'enfermement social, voire à la disparition pure et simple: la mort. Leur destinée est le thème d'innombrables livrets d'opéra et de scénarios de films muets.

Je ne parlerai pourtant pas des adaptations cinématographiques d'opéras, ce qui me conduirait à faire une analyse sur les rapports entre les arts ${ }^{1}$. Je signale tout de même que ces adaptations ont été nombreuses, notamment en Italie, pays par excellence du "mélodrame», au double sens du terme. En effet, bien des films muets sont inspirés, sinon directement par les opéras, du moins par leurs versions théâtrales ${ }^{2}$. Malheureusement, s' il est possible d'étudier les mouvements des corps des actrices du cinéma muet, les voix et les gestes des chanteuses d'opéra du passé se sont effacés : les images qui en restent sont figées, et nous n'entendons plus désormais que des cantatrices, sinon contemporaines, du moins récemment venues à la scène. Je parlerai donc surtout des personnages qu'incarnaient les protagonistes d'opéras contemporains des films. En revanche, le traitement réservé au corps de l'actrice par le cinéma permet d'imaginer comment ces héroïnes légendaires ont pu être représentées sur scène au début $\mathrm{du} \mathrm{XX}^{\mathrm{e}}$ siècle. Certes, comparaison n'est pas raison, mais si le nom de "diva» a été adopté en Italie pour qualifier les actrices du cinéma muet, on peut penser que celles-ci évoquaient non seulement la célébrité, mais également l'allure des cantatrices. Je m’interrogerai donc surtout sur les images lumineuses de femmes adulées, mais victimes de "l'idolâtrie" même qu'on leur voue (Michaud 1991, p. 125-145). Comment se construit la double forme (opératique et visuelle) du stéréotype, lui-même issu de modèles socioculturels? Comment l'écran muet représente-t-il l'éclat sonore du féminin, à travers son image charnelle, transposée en ombres sensuelles? Que se passe-t-il lorsque disparaît l'un des deux modes d'expression corporelle du féminin, lorsque la voix inaudible est évoquée à travers la mise en scène d'un corps sexué - de la gorge, du sein, de la chevelure?

Pour éviter de trop généraliser, j'ai choisi d'étudier plus spécifiquement l'Italie et trois personnages féminins de l'opéra, 
protagonistes d'œuvres contemporaines des années d'or du cinéma muet (entre 1896 et 1926). J'ai choisi les héroïnes de trois opéras de Giacomo Puccini: Tosca (1900), Madame Butterfly (1904) et Turandot, un opéra inachevé à la mort de Puccini en 1924, mais qui fut tout de même représenté en 1926. En ce qui concerne le cinéma, mon choix s'est porté, parmi les cent dive Italiennes de la même époque (glorifiées dans le beau film de Peter Delpeut, Diva dolorosa, 1999), sur celle qui demeure sans doute encore la plus célèbre parce qu'elle a su, jusqu'à la toute fin de sa vie (1985), gérer son image: Francesca Bertini, née en 1888 , et dont l'essentiel de la carrière s'est déroulée entre 1910 et 1921 .

\section{Les voix et la mort}

L'opéra est une affaire de femmes. Non, pas une version féministe; non, pas une libération. Tout au contraire: elles souffrent, elles crient, elles meurent, c'est là aussi ce qu'on appelle chanter. Elles s'exposent, décolletées jusqu'au cœur, luisantes de larmes, au regard de ceux qui viennent jouir de leurs supplices feints. Pas une qui en réchappe, ou si peu... (Clément 1979, p. 24).

Ces propos pourraient s'appliquer également à de nombreux films muets: les femmes n'y chantent pas, mais elles y meurent tout autant. Les héroïnes de Puccini sont emblématiques de ce sort cruel. La plus révolutionnaire d'entre elles, et la plus cruellement vaincue, est Tosca. L’opéra homonyme fut composé entre 1894 et 1899 et joué pour la première fois à Rome en janvier 1900, avec Eraclea Darclée dans le rôle-titre. Son canevas est tiré d'une pièce de Victorien Sardou, qui fut jouée notamment par Sarah Bernhardt (une autre diva, au théâtre cette fois). L'intrigue est composée d'événements extrêmement brutaux: le chef de la police, Scarpia, utilise la naïveté et la jalousie de la cantatrice Floria Tosca pour réussir à arrêter un révolutionnaire échappé de ses prisons. Il la désire et, pour l'obtenir, fait arrêter et torturer son amant, le peintre Caravadossi, laissant croire à Tosca qu'elle sera libre de partir avec lui. Dégoûtée par le viol qu'elle a subi, Tosca tue Scarpia. Caravadossi est toutefois 
exécuté et la cantatrice se jette du haut du Castel Sant'Angelo. Tout est réglé par l'intrigue mélodramatique: la femme qui se veut libre ne peut être que piégée; elle ne cesse, sans le savoir, de dénoncer son amour en l'appelant à tort et à travers "Mario! Mario!». De plus, elle finit par faire confiance à Scarpia, le détenteur du pouvoir, croyant ainsi s'en débarrasser. Emblème de la femme passionnée et imprudente, Tosca est également liée à une figure mythique de l'histoire: celle des révolutionnaires du $\mathrm{XIX}^{\mathrm{e}}$ siècle, épris à la fois, à la manière romantique, d'art et de liberté. En effet, Floria chante et son amant, Caravadossi, peint; tous deux soutiennent un partisan de l'éphémère république romaine, après la victoire de la réaction en 1799. Ce romantisme révolutionnaire s'exprime explicitement dans le thème de l'aria la plus célèbre de l'opéra: Vissi d'arte, vissi d'amore.

Madame Butterfly, quant à elle, représente la figure plus traditionnelle de la femme soumise. Le livret de Madame Butterfly est composé d'après une pièce de David Belasco (ellemême adaptée d'une nouvelle de John Luther Long, écrite en 1897), qui triompha aux États-Unis et que Puccini vit à Londres en 1900. Musicalement sensible à l'orientalisme, alors très en vogue (sensibilité dont on peut également trouver des marques dans Turandot), le compositeur est séduit par l'histoire de cette jeune Japonaise abandonnée par son amant américain. Écrit entre 1901 et 1903, l'opéra fit un four lors de sa première représentation ${ }^{3}$. Après plusieurs remaniements, Madame Butterfly obtint cependant très vite la faveur du public, et reste un monument du répertoire puccinien. Dans Madame Butterfly, Pinkerton, un officier de marine américain stationné au Japon, machiste et plein d'orgueil impérialiste, épouse la ravissante geisha Cio-Cio-San, qu'il surnomme Butterfly. Peu après le mariage, Pinkerton part en mer. La partie centrale du récit raconte l'attente de Butterfly qui, entre-temps, a donné naissance à un garçon. Lorsque l'officier revient, accompagné par sa véritable épouse (Américaine, comme il se doit), il veut récupérer son fils. Au moment de le lui rendre, Butterfly se fait hara-kiri, accompagnant son geste d'un air déchirant dans lequel elle dit adieu à l'enfant, qui ne comprend rien. La mort de ce délicat papillon est annoncée dès le début de l'opéra par le 
truchement de la musique (le thème final du suicide apparaît dès le premier acte, au moment où Butterfly apporte dans la maison des nouveaux mariés le sabre de son père, qui s'est luimême suicidé). À la différence de Tosca, Butterfly est une figure de victime absolue, condamnée à l'attente: l'air le plus célèbre de l'opéra, Un bel dí vedremo, exprime avec une grande pureté l'espoir qu'entretient Butterfly de voir l'infidèle revenir, et conforte le spectateur dans son impression que la jeune femme se nourrit d'illusions (elle offre un amour éternel à un imbécile, et qu'elle finisse par en mourir paraît inévitable).

La plus mythique, enfin, de ces héroïnes, est Turandot, la princesse glaciale et cruelle, vaincue par l'amour (pas le sien, d'ailleurs, mais celui qu'une autre lui donne en exemple par son sacrifice). Le livret est composé d'après une pièce du XVIII ${ }^{\mathrm{e}}$ siècle, due à Carlo Gozzi et adaptée par Friedrich von Schiller, de qui les librettistes Giuseppe Adami et Renato Simoni s'inspirèrent. Turandot fut chanté pour la première fois en avril 1926 par Rosa Raisa, sous la direction de Toscanini, qui fit arrêter la représentation là où Puccini avait, en mourant, abandonné la composition $^{4}$. Turandot incarne le mythe de la femme nocturne, liée à la lune, rebelle par son refus de l'homme, et férocement cruelle - mythe qu'on trouve dans toutes les cultures, des GrécoRomains (Diane) aux Amérindiens (pour lesquels la lune représente la tête coupée d'une jeune fille refusant le mariage). Ici, le mythe est personnifié par une princesse mandchoue, qui fait couper la tête à ses prétendants lorsqu'ils ne savent pas répondre aux trois énigmes qu'elle leur pose. Sorte de reine de la nuit, contrôlée par l'irrationnel, cette sphinge sera vaincue par un prince inconnu qui, en résolvant les énigmes, pourra l'épouser. Cependant, cela n'aura pas lieu sans que la féroce ait provoqué le suicide de la tendre Liu, une jeune esclave amoureuse du prétendant. Deux éléments s'imposent ici à notre attention. D'abord, les raisons que l'inaccessible Turandot invoque pour garder sa pureté: elle est habitée par l'âme d'une de ses aïeules, la princesse Lou-Sing, jadis violée et tuée par un brutal Mongol (la première aria de la princesse est le récit de ce viol et de ce massacre). Turandot est à la fois figure de la résistance féminine à la violence sexuelle qui hante les femmes 
de tous les siècles et, évidemment, figure mythique. L'archétype féminin s'articule à travers la mise en parallèle de la sombre princesse et de la lumineuse esclave qui, elle, aime et se dévoue jusqu’à se donner la mort, afin de ne pas être forcée de révéler sous la torture le nom du prince inconnu (et, accessoirement, parce qu'elle a compris que ce dernier, fasciné par Turandot, ne l'aime pas). Le mythe ainsi renouvelé est celui, ancestral, de la double face du féminin.

D’autres auteurs que Clément ont souligné la complexité des rôles féminins à l'opéra: " [...] à l'opéra, savoir qui est victime et qui est bourreau est plus complexe qu'il n'y paraît" (Seydoux 1984, p. 16). D'une part, les trois personnages dont on vient d'esquisser les portraits, situés au centre de l'œuvre de Puccini, ne représentent pas toutes les figures du féminin de l'opéra, qui nous ont laissé bien des images plus légères. Par ailleurs, les héroïnes de Puccini sont loin d'être unidimensionnelles, et, à l'exception peut-être de Butterfly, on ne peut considérer qu'elles ne sont que des victimes. C'est que Butterfly est un personnage plus typiquement mélodramatique que les autres: trompée et vaincue dès le départ, toujours sous la domination masculine, elle est constamment en position d'attente. En revanche, Tosca est une sorte de mise en abyme de la diva, puisque dans l'intrigue son personnage est une cantatrice (d'ailleurs, le deuxième acte met en scène la Tosca en représentation: on la voit sur scène chanter une cantate). Elle $a$, de la diva, le professionnalisme et la beauté fatale aux hommes - trois d'entre eux meurent à cause d'elle! - , beauté qui lui sera fatale à elle aussi. Quant à Turandot, c'est la figure la plus transcendantale, qui donne à la diva toute sa grandeur; cruelle, hautaine, inaccessible, elle sera pourtant vaincue par l'amour de Calaf. À elles trois, elles évoquent la complexité des désirs et des peurs qui, à travers l'art lyrique, sous-tendent la représentation du féminin.

Quelles que soient les formes des mises en scène (et elles ont été innombrables) qui tentent de jouer des corps des cantatrices et des éclairages, c'est d'abord et avant tout la musique qui produit l'effet affectif, à travers les voix qui traduisent la déception et le désespoir. Les grandes arias finales sont toutes des chants de mort: celui de Butterfly qui dit adieu à son fils avec le 
sabre déjà planté dans le ventre sous son kimono ; celui de Tosca qui se jette du haut du Castel Sant'Angelo; celui de Liu qui se sacrifie par amour. Turandot elle-même, vaincue, restera muette chez Puccini, puisqu'il n'a pas réussi à écrire son grand duo avec le prince Calaf, et qu'un autre musicien terminera à sa place l'opéra inachevé. Pourtant, en plus de la douleur, constamment exprimée par la mélodie, ni l'expression de la violence, ni celle du désir de vengeance (c'est-à-dire, en fait, de la capacité d'abstraction) ne manquent: voix coupante de Tosca tuant Scarpia ("muori, muori»), voix quasi inhumaine dans les hauteurs chez Turandot, racontant l'histoire de son aïeule assassinée (aria de la princesse Lou-Sing). Et ces trois femmes imaginaires, modelées par le drame et le chant, possèdent trois des traits dominants de la diva du cinéma muet : à la fois belle et passionnée à en mourir, inaccessible et trop humaine, celle-ci est fondamentalement dangereuse - pour les autres et pour ellemême. Comment les actrices condamnées à l'image muette réussissent-elles à exprimer ces affects extrêmes et fondamentaux? Comment se fait la transposition de la scène où résonnent les voix à l'écran muet?

\section{La Bertini : une carrière d'actrice}

Au moment même où l'on joue en Italie les opéras de Puccini, se développe la carrière de Francesca Bertini, considérée comme un parangon de la diva italienne, et dont la figure est relativement complexe. Après avoir fait ses débuts comme actrice de théâtre, elle est rapidement remarquée en 1908 par la société Film d'Arte grâce à son jeu dans la pièce de Salvatore di Giacomo, Assunta Spina. Elle a alors à peine vingt ans. Engagée d'abord pour deux ou trois rôles secondaires, notamment dans Salomé, elle occupe rapidement le devant de la scène et interprète en deux ans les rôles principaux d'une vingtaine de films courts, tirés de la littérature classique, du théâtre et de l'opéra: Francesca da Rimini (inspiré de La divine comédie), Béatrice d'Este, ou encore Tristan et Isolde et Roméo et Juliette. La diversité des protagonistes qu'elle incarne ensuite ne la cantonne pas dans le registre tragique ou mélodramatique: en 1912, par exemple, elle joue aussi bien dans un film à grand déploiement, Rose de 
Thèbes, réalisé par Ernesto Guazzoni, que dans un film à tendance réaliste, Il fascino della violenza, ou encore dans une comédie, Panne d'auto, où deux automobilistes se disputent son amour. En 1914, elle sort même tout à fait de ses rôles habituels avec Histoire d'un Pierrot ${ }^{5}$, dans lequel elle se donne des airs ambigus sous son déguisement androgyne, abandonnant ainsi les poses classiques.

Sans entrer dans le détail, on peut souligner la diversité des films à succès dans lesquels elle joue ensuite (entre autres: Nelly la gigolette, d'Emilo Ghione, 1914; Diana l'affascinatrice, de Gustavo Serena, 1915; Tosca, d'Alfredo De Antoni, 1918, "inoubliable» selon la critique), ainsi que sa prestation dans la série des "Sept péchés capitaux", réalisés en 1918-1919 (de La gourmandise, de Camillo de Riso, à La luxure, d'Eduardo Bencivega, en passant par L'avarice, de Gustavo Serena). Elle se lance alors dans la production, avec la Bertini-film, activité qu'elle poursuivra jusqu'en 1925. Toujours appréciée de la critique pour l'élégance de sa silhouette et la beauté plastique de son visage, elle l'est aussi pour son caractère protéiforme, et ce malgré quelques fausses notes: «[...] il y a sept péchés capitaux, le $8^{e}$ a été commis par la Bertini-film et la Cæsar-film, en s'en inspirant pour une série de films interprétés par Francesca Bertini» (cité dans Mingozzi 2003, p. 161). Elle remplit encore les salles avec un grand nombre de films sortis entre 1920 et 1922, mais se retire dès 1921 (pas complètement, cependant), évitant ainsi de perdre une aura qu'elle saura conserver dans les années 1930 et 1940, après l'arrivée du parlant. Très belle, certes, mais aussi très professionnelle, experte dans l'art des entrevues, tenant dans la vie à la fois son rôle d'actrice admirée et celui d'une femme de tête, elle sait contrôler ses photos, les articles qu'on lui consacre (notamment dans la presse féminine italienne) et, pour une part, ses films. Comme toutes les divas ou, ailleurs, les stars, elle est un "produit», mais elle est surtout son propre "produit", grâce à l'intelligence et au talent avec lesquels elle construit sa propre image, comme on le voit dans les interviews qu'elle donne encore dans le film L'ultima diva, de Gianfranco Mingozzi, en 1982. 


\section{Le travail du corps}

Assunta Spina, en 1915, permet à Bertini d'atteindre le sommet de sa carrière. Elle y incarne le rôle le plus important de sa vie, depuis ses premières représentations théâtrales jusqu'à cette sorte de répétition générale qu'a été en 1912 Il fascino della violenza, de Baldassare Negroni, où elle endossait le rôle de la "femme du peuple» qui se sacrifie après avoir provoqué un meurtre par jalousie. Assunta Spina, adaptation de la pièce de Salvatore Di Giacomo, est officiellement dirigé par Gustavo Serena, qui écrit le scénario en collaboration avec Bertini. L'actrice revendique néanmoins haut et fort sa participation à la réalisation, que reconnaît Serena: "Dans un parfait dialecte napolitain, elle organisait, commandait, disposait les comparses, le point de vue, l'angle de la caméra; si elle n'était pas convaincue par une scène, elle prétendait la refaire une seconde fois" (Mingozzi 2003, p. 152). C'est son film. Et son triomphe, d'ailleurs: le film est encore aujourd'hui reconnu comme un chef-d'œuvre, comme il le fut tout au long des années 1920.

Elle y interprète le rôle-titre, celui d'une belle et séduisante jeune popolana napolitaine, courtisée par Michele, qui l'engage comme blanchisseuse, et par Raffaele, avec qui elle fait un peu trop la coquette, notamment le jour de son anniversaire. Furieux, Michele la blesse d'un coup de couteau au visage; ensuite arrêté, il est condamné à deux ans de prison. Le greffier $\mathrm{du}$ tribunal tente alors sa chance auprès d'Assunta, et elle finit par lui céder. Libéré plus tôt que prévu, le soir de Noël, Michele tue ce nouveau rival et, à l'arrivée de la police, prise de remords, Assunta s'accuse du meurtre. Il est clair que l'on nage dans les stéréotypes machistes: alors que l'homme (Michele) reste sympathique malgré sa violence, puisqu'il est jaloux (avec ou sans raison), Assunta est coupable de distribuer trop largement ses sourires: cela l'entraîne vers la quasi-prostitution, puis vers l'autodestruction.

Les vagues ressemblances entre les thèmes d'Assunta Spina et ceux de Carmen (1875) de Georges Bizet - une superbe femme du peuple, prête à toutes les coquetteries; la prophétie d'une zingara, qui annonce du sang comme les cartes annonçaient la mort à Carmen — sont accentuées par un ajout, proposé par 
Bertini elle-même, au scénario original: son personnage séduira, sans même le vouloir, le carabinier chargé de l'arrêter. Ce sont surtout les postures et la gestuelle d'Assunta (en fait, de Bertini) qui évoquent celles de Carmen, telles qu'elles sont traditionnellement représentées: dans ce film, le jeu corporel a une efficacité qui tient à sa simplicité symbolique, inspirée de l'opéra, où la nécessité de chanter des arias immobilise souvent la cantatrice, la tête portée très haut, la gorge exposée (du moins à cette époque). Le corps rejeté en arrière, les poings souvent placés sur les hanches, dans un geste d'affirmation ou de révolte, ou les bras tendus ou levés, dans un geste de douleur ou de supplication, la tête haute, la gorge dégagée, sont typiques du jeu des divas italiennes. L'un des talents de la Bertini fut d'exploiter cette gestuelle au maximum, tout en sachant en inventer des variantes.

On peut en juger en observant l'actrice dans ses poses les plus célèbres, reproduites sous forme d'images photographiques (en Marguerite Gauthier, 1915 ; en Tosca assassinant Scarpia, 1918), et encore plus directement perceptibles dans les films restaurés (on a pu en voir plusieurs à Bologne, en 2003, au festival Il Cinema Ritrovato). Dans la scène où Assunta Spina se présente au tribunal, le châle et la jupe qu'elle porte sont les instruments dramatiques qui - tout en évoquant le personnage de Carmen - permettent de traduire, dans leurs mouvements autour du corps de l'actrice, le caractère séduisant et vulnérable à la fois du personnage stéréotypé de la diva (figures 1 à 4). Le corps de l'actrice, en imitant les poses de la cantatrice, traduit ce que la voix exprime à l'opéra, sans doute en l'exagérant. Comme le dit Francesco Pitassio (2002, p. 196-197), le "divisme» est caractérisé par un "corps excessif ». Un autre historien italien, Michele Canosa (1997, p. 19), précise qu' «il ne s'agit pas de jouer un rôle, d'incarner un personnage. C'est un corps qui tremble, qui se tord, qui tient la pose. C'est un corps possédé». Puisque l'absence de son empêche la diva du cinéma muet de jouer, comme à l'opéra, sur la voix, ou, comme au théâtre, sur la diction, c'est son corps qui remplace l'une et l'autre. Il devient ainsi une composante esthétique majeure du cadre, comme le sont, sur les affiches, les jeux de lumière sur les étoffes et les 


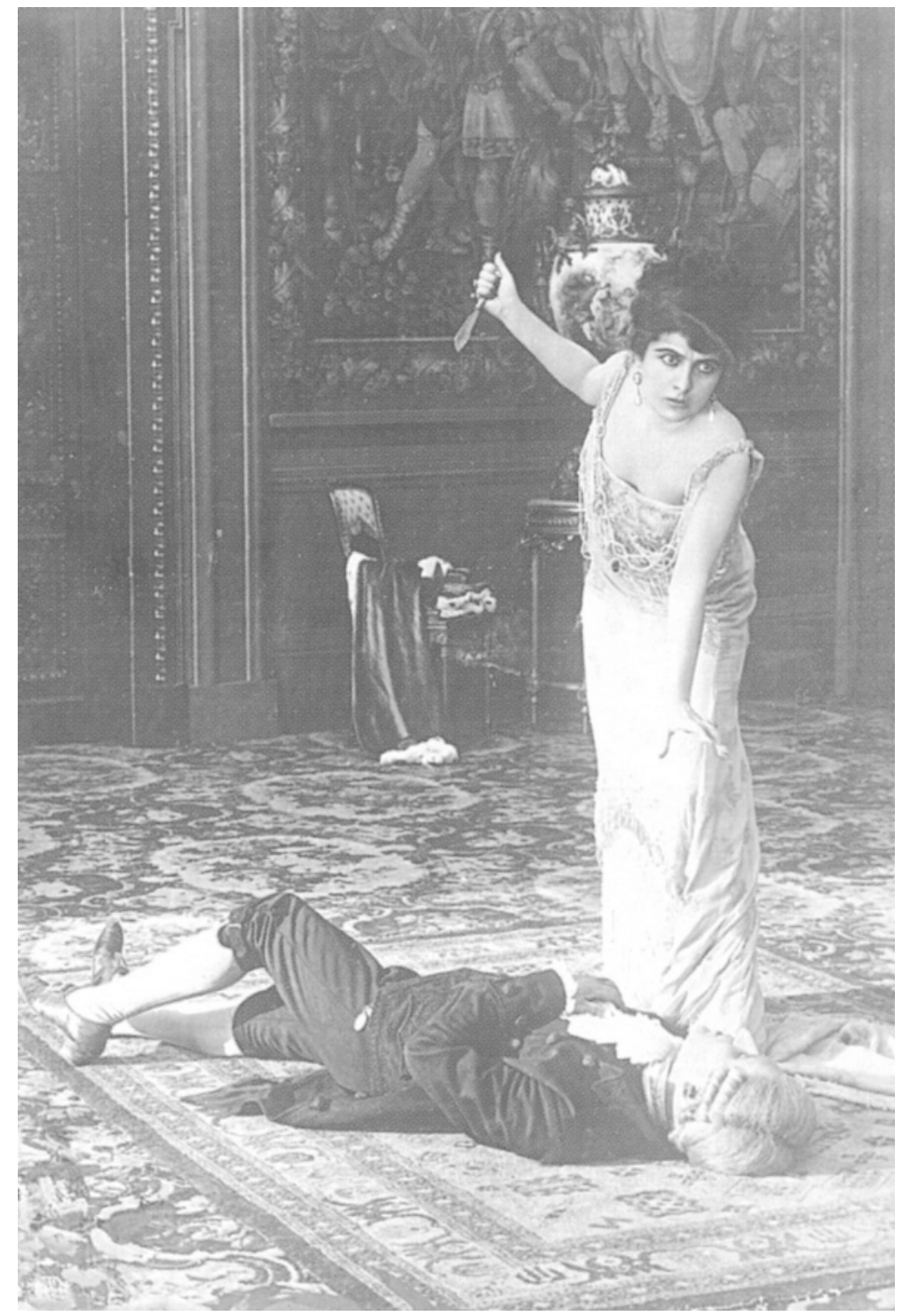

Figure 1. Tosca, ou la pose de la diva d'opéra sur scène (Tosca, 1918) (C) Archivio fotografico, Cineteca di Bologna

visages des femmes dessinées par Alphonse Mucha ou Aubrey Beardsley. Dans les films muets italiens mettant en vedette des divas, la composition du plan, où la façon dont le corps est cadré est déterminante, donne aux "divines» toute leur puissance. 


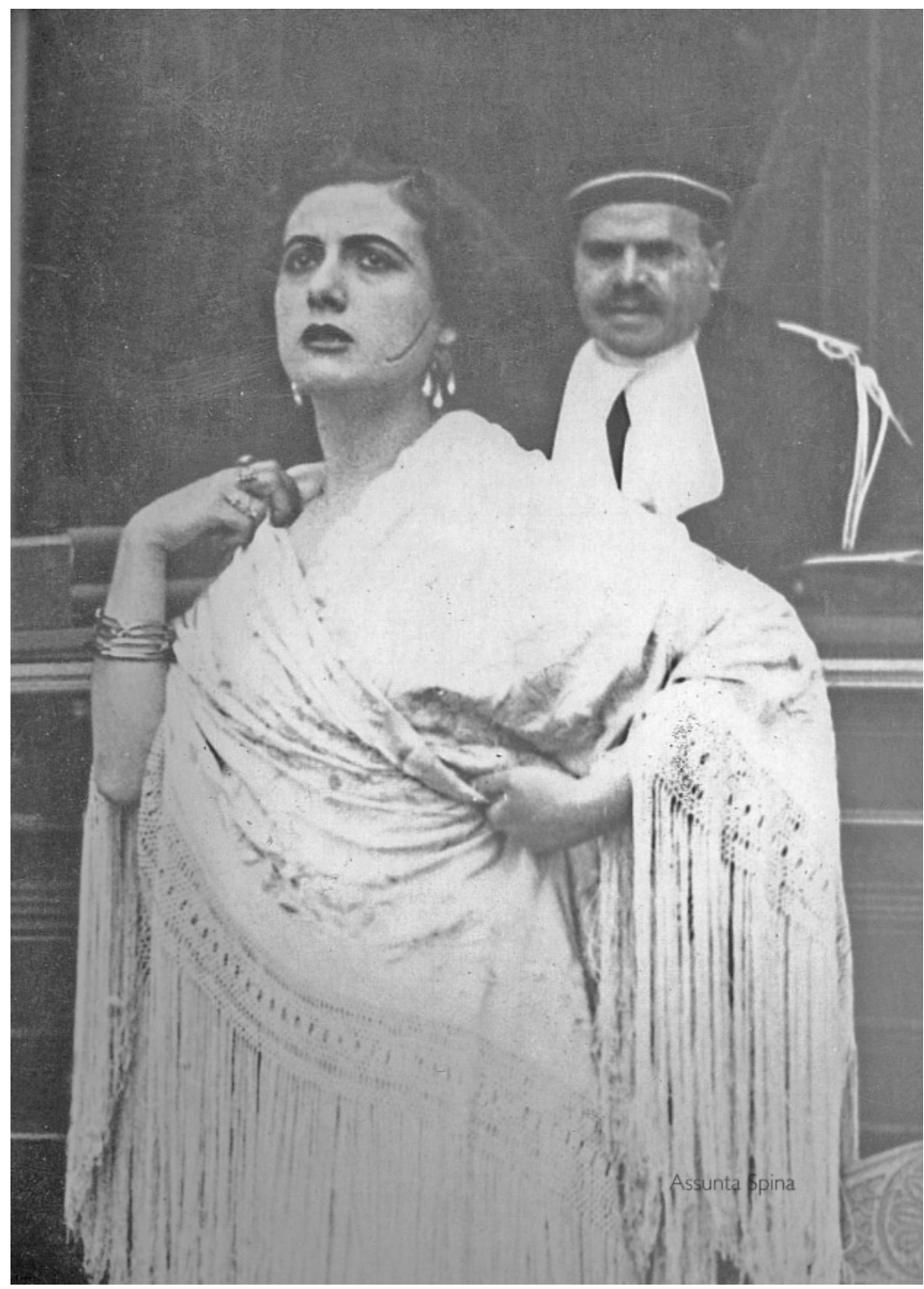

Figure 2. Bertini: Assunta ou Carmen? (Assunta Spina, 1915) (C) Archivio fotografico, Cineteca di Bologna

La gorge et les yeux; les jeux de la lumière

La silhouette élégante, les vêtements éclatants, les écharpes, châles et autres voiles sont d'indispensables accessoires scéniques de l'actrice-diva, au cinéma comme sur la scène d'opéra, mais, à la différence de la scène (de théâtre ou d'opéra), où le spectateur 


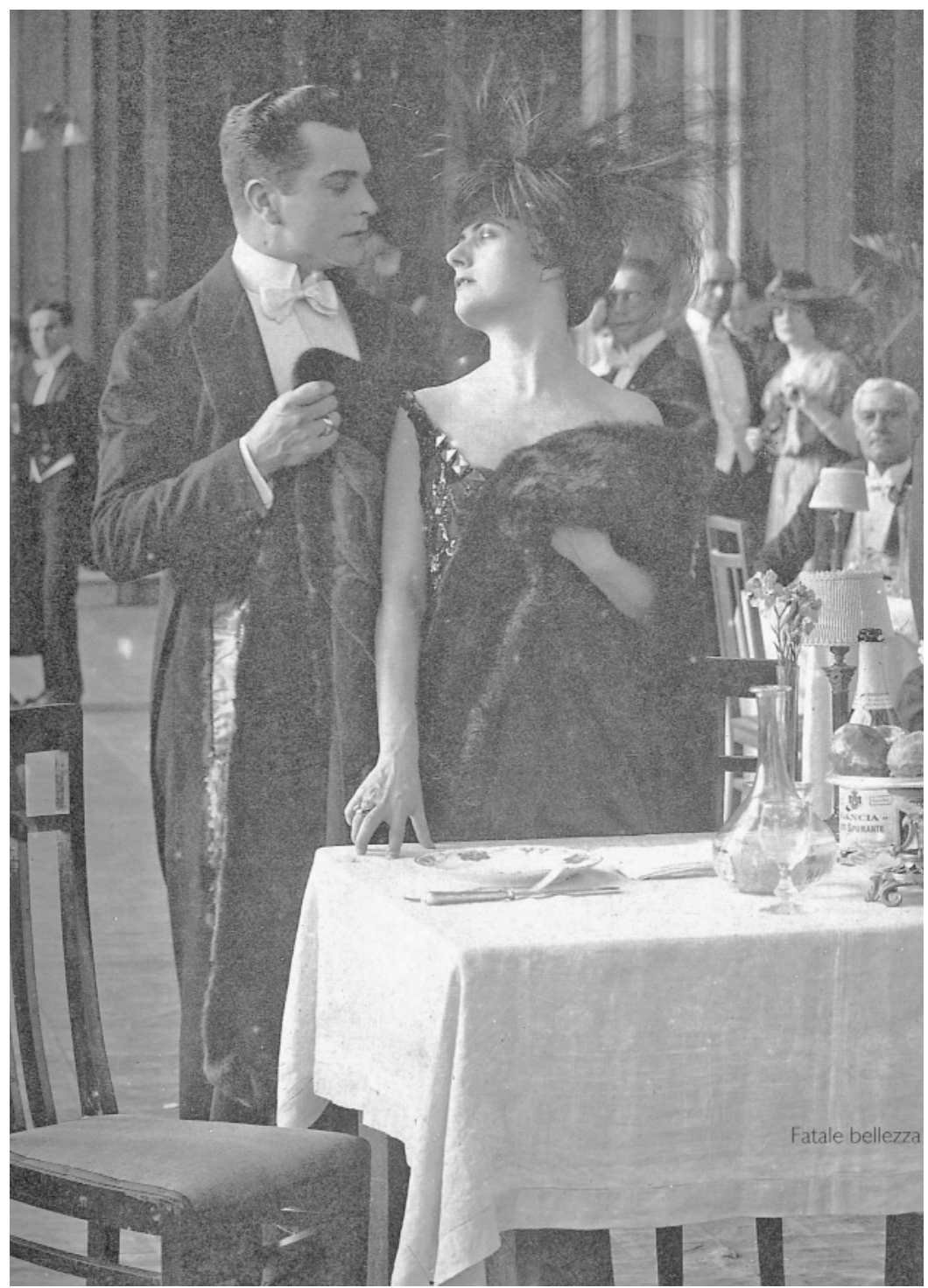

Figure 3. La gorge et le regard: fatale beauté (Bellezza Fatale, 1919) (C) Archivio fotografico, Cineteca di Bologna

est trop éloigné pour avoir autre chose qu'une vision d'ensemble, l'écran permet la proximité, l'intimité avec le corps même de l'actrice, en tant qu'icône du féminin, et ce tout particulièrement dans les plans qui insistent sur la gorge et le visage. Il ne s'agit plus ici seulement de la beauté ni de la 


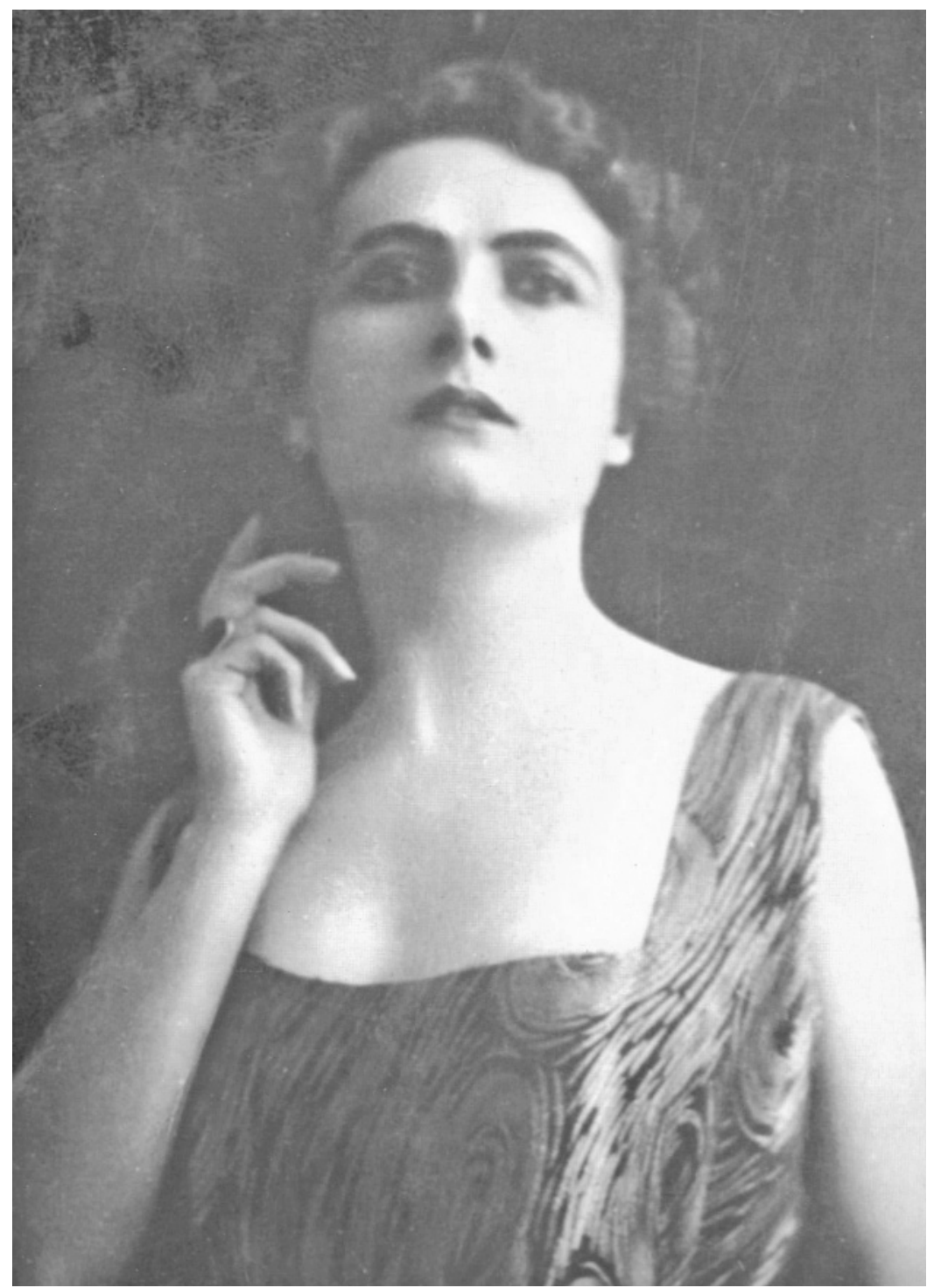

Figure 4. La magie du visage; portrait de Francesca Bertini (C) Archivio fotografico, Cineteca di Bologna

gestuelle ou des mimiques expressives, mais de la manière dont l'art du cinéma les magnifie. Trois armes sont essentielles pour cela: le jeu des regards, le gros plan et l'éclairage.

Le «lieu» principal du corps est la gorge, d'où provient la voix et qui permet le raccord entre le tout (le corps) et le détail à 
valeur symbolique (le visage), qui, lui, va exprimer les sentiments. On le voit lors d'une scène de séduction impérieuse, dans Fatale beauté (Gaston Ravel, 1922 ${ }^{\circ}$ ), où la Bertini s'affirme en vamp dangereuse (comme dans un portrait de Carlo Siviero, offert par Bertini elle-même à la Cinémathèque française) : l'éclairage est concentré sur la gorge de l'actrice, qui apparaît, si l'on peut dire, comme le «cœur» du corps et de la sensibilité. D'où les décolletés vertigineux, les têtes haut levées et les mentons en l'air. D'où, aussi, l'impression de fragilité qui se dégage souvent des personnages de femmes, en contradiction avec la dignité, voire l'arrogance ou la morgue dont leurs manières témoignent: ces seins, ces gorges, sont exposés à tous les coups possibles, qui mettent le féminin en perpétuel danger. Les actrices aussi, d'ailleurs, semblent constamment en danger: la brièveté de leur carrière ne tient pas seulement au passage au parlant et à la maîtrise de la voix, que toutes n'avaient pas, mais également, très concrètement, à la nécessité d'avoir l'angle de la mâchoire parfait, le menton et le cou toujours impeccablement tendus, donc de ne pas vieillir.

Les postures que les divas italiennes adoptaient dans leurs films expliquent aussi l'orientation particulière de leurs regards. Selon Jacques Aumont (1992, p. 55), "le regard du visage ordinaire [au cinéma] est pris dans un jeu interminable, comparable seulement à la circulation des paroles». Mais, si le jeu des échanges de regards est important au cinéma en général, il constitue l'un des caractères les plus remarquables des films des divas italiennes. Souvent, le regard de la diva passe au-dessus de la tête du spectateur, perdu dans un lointain indécis, ce qui lui donne l'air de se porter vers des lieux éthérés, hors des contingences humaines, là où précisément nous entraîne la voix des cantatrices. Les effets de regards n'excluent pas, en particulier chez les grandes actrices comme la Bertini, le rôle des mimiques, qui permettent de faire passer l'affect, les sentiments, l'émotion, et qui sont essentielles pour le cinéma muet. Le visage muet est un "visage immédiat [...]. Il se donne tout entier et d'un coup, il s'offre à l'intuition, non au déchiffrement" (Aumont 1992, p. 78) —, comme la voix de la chanteuse d'opéra. Grâce aux expressions des divas du muet, en particulier lorsqu'elles sont 
filmées en plan serré ou en gros plan, nous saisissons directement l'enjeu d'une scène, les rapports et les "accords" (au sens musical du terme) de sentiments variés. C'est en cela que les divas du cinéma italien et de l'opéra se ressemblent. Cependant, le visage de la cantatrice reste toujours assez peu visible pour le spectateur, et n'occulte donc pas le personnage; le visage de la diva de cinéma, quant à lui, est toujours (malgré le maquillage) reconnaissable, effaçant ainsi à son profit les particularités du personnage. Cela finit par donner l'impression que les différents personnages qu' interprète une actrice se ressemblent.

Le visage de la diva n'est pas qu'un visage ordinaire, c'est un visage-tableau: maquillé, composé, éclairé. Yeux et bouche sont accentués, certes, mais une attention particulière est accordée au travail de la lumière, à la photographie, aux effets de flou ou de lumière frisante qui donnent aux traits, comme à la pose, une valeur à la fois abstraite et dramatique, produisant l'effet d'un visage peint. Parfois, ces effets sont accentués par différents "jeux de miroirs", dans lesquels les images du féminin se trouvent enfermées. Par exemple, dans le film Frou-Frou (Alfred De Antoni, 1918), l'actrice change trente fois de tenue en une heure, effectuant, comme un mannequin, une véritable

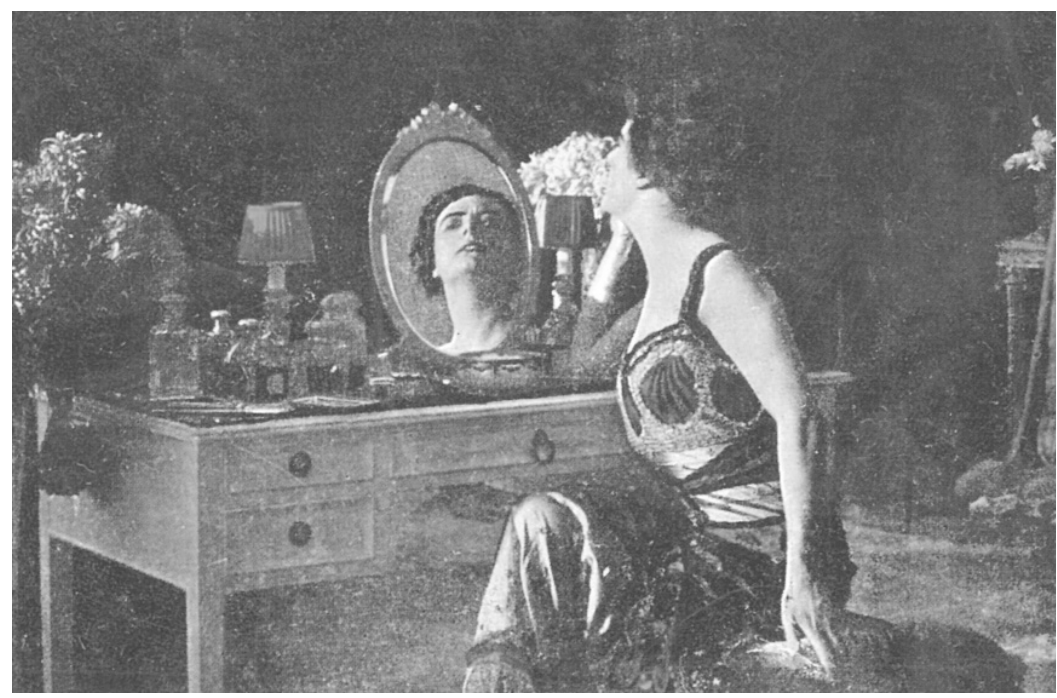

Figure 5. Le jeu du reflet : entre portrait et miroir (Frou-Frou, 1918) (C) Archivio fotografico, Cineteca di Bologna 
présentation de la collection du couturier Paquin. Les jeux de miroirs peuvent aussi permettre d'exprimer la complexité d'un personnage aux sentiments contradictoires, comme dans Andreina (Gustavo Serena, 1917), où la Bertini incarne une ingénue dont les différents «reflets" mettent en évidence un monde faux et hypocrite.

\section{Fracture}

Faisant référence à Louise Brooks, Leonardo Quaresima (1981, p. 9) soulignait à quel point la construction du corps excessif de la star, si éclatante soit-elle, joue un rôle normalisateur, parce que cette construction a pour effet de "régler» le corps; ainsi l'industrie cinématographique "standardise-t-elle» les scandaleux excès sociaux mis en scène dans les mélodrames, en transformant les figures de la déviation et de la subversion en symboles d'unification et d'alignement. Dans le cas de Louise Brooks, il s'agit d'une actrice au service d'un "cinéma institutionnel », qui a déjà "réglé» la figure de la star hollywoodienne. En revanche, avec la diva italienne, moins industrialisée, un paradoxe est mis en évidence, trace d'une faille dans la construction de la norme. Certes, cette femme portée par la gloire n'est pas autre chose qu'une figure imaginaire, enfermée dans des conventions comme elle l'est dans le silence. Quels que soient sa beauté et son éclat, elle est forcément vouée à se taire. Mais alors que tout, dans les intrigues mélodramatiques, montre la femme vaincue, défaite, tout, au contraire, dans la posture, la gestuelle, la composition du visage, les jeux de lumière, dit sa grandeur sublime, celle d'un corps qui serait la "prothèse" d'une voix. Tout se passe comme si, dans ses représentations, la femme ne pouvait être à la fois un corps et une voix.

Personne mieux que Visconti n'a su montrer l'importance de cette fracture entre la voix et le corps, bien qu'il ait travaillé longtemps après le muet et monté des opéras plus verdiens que pucciniens. Ainsi, dans la première séquence de Senso, il met en valeur les symboles mêmes du féminin cinématographique, filmant d'abord la comtesse Livia devant son miroir (son cou, ses épaules, la naissance de ses seins), puis nous la montrant ensuite violée par le regard concupiscent du lieutenant Mahler. 
En mettant en parallèle sa gorge avec la voix de Léonora, personnage du Trouvère dont le corps rigide et empoté n'exprime rien, il fait de la comtesse Livia le double charnel de la voix de la cantatrice. En ajoutant à la nouvelle de Boito qui a inspiré le film un prologue qui n'existait pas, Visconti célèbre la connivence du cinéma et de l'opéra, et construit une "femme imaginaire» et condamnée. Piégée par sa sensualité, victime de la lâcheté masculine, Livia se perdra en trahissant la révolution d'abord, son amant ensuite, et disparaîtra finalement dans une mort d'autant plus ignominieuse qu'elle ne nous est même pas montrée. Elle est la figure même de la condition féminine, donc toujours vaincue. Mais elle incarne aussi une figure renouvelée des divas du muet, qui ont su rendre la dimension mythique de la femme d'opéra par l'usage de postures et d'une gestuelle spécifiques, et surtout en nous offrant cette gorge dénudée et ce visage expressif, magnifiés par le maquillage et l'éclairage.

\section{Que reste-t-il des divas?}

Ce rapport étroit entre les poses de l'opéra et celles des actrices du muet, dont la trace persiste dans plusieurs films de Visconti, est très spécifiquement italien, et traduit à quel point l'opéra, mais aussi les films muets, ont marqué le paysage culturel de l'Italie (du moins jusqu'au dernier tiers du XX $\mathrm{XX}^{\mathrm{e}}$ siècle). Par ailleurs, l'étrange confusion dans le passage de la scène à l'écran, qui s'exprime de manière exemplaire dans ce plan de Senso où Livia (la femme "réelle» du film, incarnée par l'actrice Alida Valli) est redoublée sur scène par l'héroïne d'opéra (Léonora, la «fausse» chanteuse, dont la voix est enregistrée), permet de mieux saisir le glissement sémantique du terme diva: de la cantatrice, dont la voix seule porte le rôle, au corps de lumière de l'actrice. À l'opéra, au moins de la Malibran jusqu'à la Callas, ce sont bien les cantatrices qui sont les divas, mais leur image personnelle (souvent celle d'une femme théâtrale et malheureuse) se confond irrémédiablement avec celle des hérö̈nes dont elles ont chanté le rôle (évidemment théâtral et malheureux). Prestige d'un art bien ancré dans la culture légitime? On joue encore les opéras sur toutes les scènes lyriques $\mathrm{du}$ monde, et chaque nouvelle grande cantatrice efface, au 
moins partiellement, les précédentes, et ce malgré les techniques d'enregistrement de la voix : c'est Tosca qu'on écoute, chantée par la Callas (ou la Gheorghiu), comme si la voix de Tosca passait d'un corps à l'autre, effaçant partiellement la personnalité de la diva, et comme si Tosca elle-même se réincarnait sous un autre nom. Au contraire, les figures de divas au cinéma renvoient davantage aux personnes qu'aux personnages, même si certains rôles leur collent à leur peau: ce sont la Bertini, la Borelli ou la Menichelli (les "trois grâces" de ce cinéma muet italien) qui ont marqué les mémoires, alors qu'à quelques exceptions près (Assunta Spina pour la Bertini, par exemple) leurs personnages ont été à peu près oubliés. Les films muets, longtemps dédaignés, ne ressortent, restaurés, que pour les happy few, plus ou moins spécialistes ou cinéphiles. La mémoire de ces divas est donc moins liée à leurs rôles qu'à une culture médiatique visuelle: le corps même de l'actrice est là, sur la bande filmique, qui en conserve la trace éclatante; photos, affiches, articles de presse du star-system naissant ont permis de les figer dans leur image de "divines", alors que les personnages qu'elles ont interprétés se sont décolorés. Le terme de diva ne s'applique plus alors à des noms jouant le rôle de support mémoriel pour des voix ${ }^{7}$, mais à des corps de lumière.

Université Paris III

\section{NOTES}

1. Pour un exemple de ce type d'étude, voir Pescatore 2001.

2. Par exemple, l'intrigue de La traviata est inspirée de La dame aux camélias, d'Alexandre Dumas fils. L'intrigue de Tosca, quant à elle, est tirée d'une pièce homonyme de Victorien Sardou.

3. Cette représentation eut lieu à la Scala en février 1904, avec Rosina Storchio dans le rôle principal. La version de l'opéra la plus couramment représentée est celle de 1906.

4. La fin de l'opéra fut composée par un autre musicien, Franco Alfano, entre 1924 et 1926.

5. Les trois derniers films mentionnés sont du même réalisateur: Baldassare Negroni.

6. Le film, tourné en 1919, dut faire quelques concessions à la censure et il fallut attendre 1922 pour pouvoir le sortir.

7. Cela vaut par exemple pour la Malibran, mais pas pour la Callas, dont la situation est plus ambiguë, Callas ayant joué au cinéma dans Médée. 


\section{RÉFÉRENCES BIBLIOGRAPHIQUES}

Aumont 1992: Jacques Aumont, Du visage au cinéma, Paris, Cahiers du cinéma, 1992.

Bertini 1969: Francesca Bertini, Il resto non conta, Pise, Giardini, 1969.

Canosa 1997: Michele Canosa, "Muto di luce», dans Michele Canosa et Antonio Costa (dir.), A nuova luce. Cinema muto italiano, Bologne, CLUEB, 1997.

Clément 1979: Catherine Clément, L'opéra ou la défaite des femmes, Paris, Grasset, 1979.

Martinelli 2001 : Vittorio Martinelli, Le dive del silenzio, Bologne/Gênes, Cineteca di Bologna/Le Mani, 2001.

Michaud 1991 : Stéphane Michaud, "Idôlatries», dans Georges Duby et Michelle Perrot (dir.), Histoire des femmes en Occident. Le XIX siècle, Paris, Plon, 1991.

Mingozzi 2003: Gianfranco Mingozzi, Francesca Bertini, Bologne/Gênes, Cineteca di Bologna/Le Mani, 2003.

Pescatore 2001: Gugielmo Pescatore, La voce et il corpo. L'opera lirica al cinema, Pasian di Prato, Campanotto Editore, 2001.

Pitassio 2002 : Francesco Pitassio, Ombre silenziose, Pasian di Prato, Campanotto Editore, 2002.

Pitassio 2003 : Francesco Pitassio, Attore/Divo, Milan, Il Castoro, 2003.

Quaresima 1981: Leonardo Quaresima, "Louise Brooks: il tulle bianco e il tulle rosa", Cinema \& Cinema, $\mathrm{n}^{\text {os }}$ 27-28, 1981.

Seydoux 1984: Hélène Seydoux, Laisse couler mes larmes. L'opéra, les compositeurs et la féminité, Paris, Ramsay, 1984. 